\title{
Improving Asia-Pacific Science Collaboration
}

Recommendations affecting researchers, curricula, institutions and tenure in the Asia-Pacific could increase innovation.

\author{
Stephen J. Toope, ${ }^{1}$ Chorh Chuan Tan, ${ }^{2}$ Nina V. Fedoroff ${ }^{34}$
}

$\mathrm{A}$ lthough there are important scientific contributions being made around the globe, the most dramatic new developments are taking place among nations in the Asia-Pacific. Of course, the United States and Japan have long been leading Asia-Pacific innovator nations in science and technology, and Australia and Canada have been solid contributors to knowledge. But it is the relatively new entrants from the

Asia-Pacific that are chang-

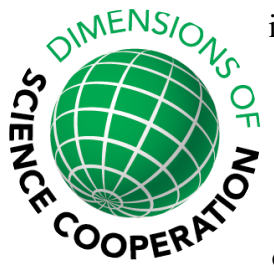

ing the dynamic of science around the globe. Most extraordinary is the rise of China. According to a Royal Society report (1), the publications output of Chinese scien-

tists is set to surpass that of U.S.-based scientists by 2013. Major investments in discovery and innovation are building capacity in Korea, Singapore, and Taiwan. Enhanced collaboration between institutions has the potential to lead to higherimpact research (2) and to tap the widening base of research expertise. Given the rapid rise in trade and economic cooperation among countries in the Asia-Pacific region, we propose that a concerted and immediate effort is required to enhance Asia-Pacific science collaboration.

Promoting Researcher-to-Researcher Linkages Over the last decade or so, great effort has been expended in trying to develop university-to-university linkages globally and around the Pacific. These forms of linkage include the Association of Pacific Rim Universities (www.apru.org); Universitas 21 (www.universitas21.com); the International Alliance of Research Universities (www. iaruni.org); and the International Association of Universities (www.iau-aiu.net).

${ }^{1}$ Professor of Law, and president and vice-chancellor, University of British Columbia, Vancouver, British Columbia V6T 1Z4, Canada. 'Professor of Medicine and president, National University of Singapore, Singapore. ${ }^{3}$ Evan Pugh Professor, Huck Institutes of the Life Sciences, Penn State University, University Park, PA 16802, USA. "Distinguished visiting professor, King Abdullah University of Science and Technology (KAUST), Thuwal 23955, Saudi Arabia.

*Author for correspondence. E-mail: stephen.toope@ubc.ca

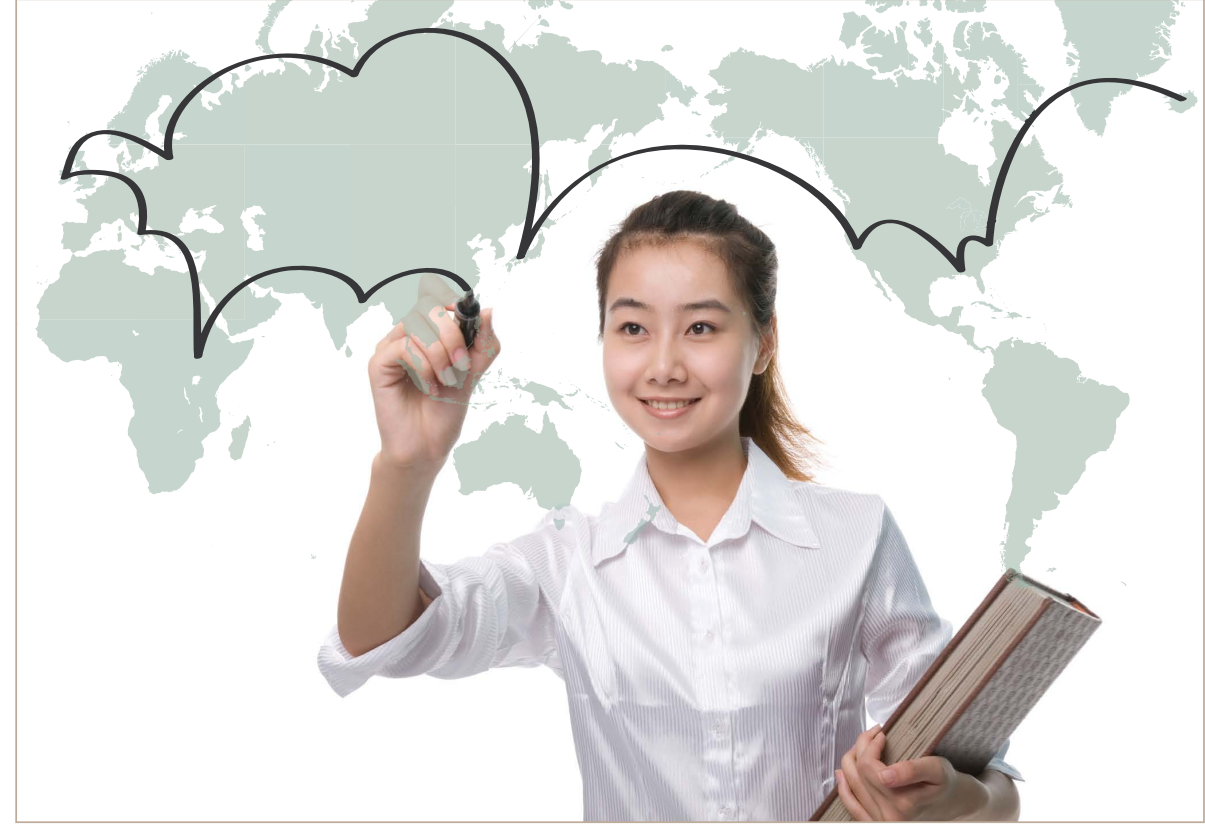

Although these networks have helped create expanded opportunities in undergraduate student mobility and in the sharing of organizational experience, none has had substantial impact in promoting enhanced research collaboration. Although seed-funding of small research initiatives and workshops has helped bring researchers together, this has not resulted in larger-scale collaborations because these efforts have been defined in a largely top-down manner, and there is a lack of adequate follow-on funding that could support collaborators across different countries. There are, however, ways of helping to encourage individual faculty and other researcher linkages through operational approaches that help identify needs, potential partners, and resources.

For example, the Global Knowledge Initiative (http://globalknowledgeinitiative. org/) has launched a series of programs that seek to connect resources and people around science, technology, and innovation challenges of the developing world. The organization is active globally, working with scientists to identify challenges, develop purpose-driven networks to tackle them, and build the skills required to innovate collaboratively. Injecting rigor into the process of locating resources and partners (rather than the commonly observed, ad hoc approach) and making sense of the context in which collaborative innovation occurs, the organization straddles the divide between national and regional development goals and individuals' capability and need to achieve them.

\section{Sharing Curricula}

One of the least efficient aspects of global university culture is the constant reinvention of curriculum at the department, and even individual faculty member, level. It is difficult for professors at the best-resourced institutions to keep up with all the developments in a teaching field that is usually wider than the professor's specific research interests. For university teachers in the developing world, the effort is virtually impossible. If we want to create a more level playing field, use our professors' time more productively, and help to create the talent that will allow more effective international collaboration to flourish, academic leaders must consider investing collectively in curricula that could be shared regionally.

The Massachusetts Institute of Technology (MIT) open-curriculum platform is admirable in concept, but is not likely to 
fundamentally reshape curricula in the Asia Pacific because it is a one-way projection. We suggest that deans and curriculum development leaders from institutions in different countries could productively work together to develop curricula for specific courses that are at once up-to-date, transferable, and sensitive to resource constraints in different settings. In parallel, efforts could be made to engage with digital innovators to see how new technologies might facilitate the wider sharing of the curricula developed.

Although language barriers are real, they are not insurmountable. English is the lingua franca of science for the time being, and it would be possible to draw together teams to develop curricula in English, which could then be translated into a range of languages for students in various countries.

Another great inefficiency in university curricula is the offering of advanced courses to small groups of students. In many fields, these courses are vital and must be taught every year, but only to the small numbers of students specializing in those areas. Simply forming consortia and teaching these courses collaboratively, perhaps using interactive digital systems, would permit departments to cut their loads while allowing students and faculty from different institutional settings and countries to learn from each other. An example is the National University of SingaporeUniversity of Toronto joint minor in environmental science that leverages on complimentary academic strengths and avoids the need to develop "duplicate" capabilities.

\section{Making International Collaboration Count in Universities}

International collaboration is an effective and powerful way of bringing complementary expertise together to pursue and achieve higher-impact science research (3). At the same time, international collaboration can also result in some cost and efficiency savings by reducing the duplication of equipment and expertise in different localities. Yet, university faculty may choose not to pursue international collaboration opportunities for a variety of reasons, including, in particular, how collaborations are evaluated for tenure and promotion. Collaborative work, especially interdisciplinary work, is often discouraged because of the view that young faculty members must establish their individual reputations in their own disciplines.

Tenure and promotion decisions are among the most important ways of influencing the future direction of universities. The balance that has been struck in most globally influential universities is to focus tenure and promotion decisions equally on research and teaching, with less but some emphasis being given to "service." We think that universities and their faculty associations would benefit from explicitly taking productive international collaboration into account in the promotion and tenure process.

We are not suggesting that every professor must collaborate internationally. However, international collaboration is appropriate in many disciplines, and especially in fields that address global challenges. Finding concrete ways within university processes to recognize and create incentives for such collaboration is important.

\section{Building Innovation Ecosystems}

For decades now, social science researchers have identified the need to build "clusters" for innovation to thrive and to be sustainable (4). Innovation "hubs" have been identified in Silicon Valley (5), along route 128 outside Boston (6), in Cambridge in the UK (7), and in Tokyo (8), just to offer a few examples. It is clear that one of the key elements in the creation of such clusters or hubs of innovation is the presence of at least one major research university. The most successful hubs have access to a collection of leading institutions of higher learning and research. But great universities alone are not enough.

To create powerful centers of scientific and technological discovery and innovation, an entire "innovation ecosystem" is required, (9) which encompasses a critical mass of diverse types of talent. These include high-quality researchers and graduate students and, in the case of biomedicine, clinician-scientists and clinical researchers linked to hospital systems that are strongly supportive of translational clinical investigation. Beyond these elements, the ecosystem needs experienced entrepreneurs who can incubate and grow promising start-up companies or can mentor others to do so. The presence of investors and venture capitalists willing to invest at various stages of development is crucial, particularly to provide early-stage funding and, in many cases, business expertise. Large technology or pharmaceutical companies, with their corporate laboratories, bring industry research and development (R\&D) perspectives to the ecosystem, as well as expertise and opportunities for collaborative research, often with specific applications in mind.

To work well, the ecosystem must foster an environment that promotes a free flow of people, ideas, and experiences across institutions and sectors. This sharing may be hampered by the traditional preoccupations with intellectual property protection, technology transfer, and commercialization at the level of individual institutions.

We therefore encourage the convening of a group of relevant organizations to consider how stronger innovation ecosystems could be created in the Asia-Pacific and, in particular, the role of enhanced transnational cooperation. The organizations should include agencies that promote science, science and technology research funders, universities, and key business groups in the Asia-Pacific region.

\section{Further Implications}

The action steps that we detail here would, we believe, help to promote much stronger scientific collaboration in the Asia-Pacific. Other steps are implied in what we have suggested. These include greater talent mobility among graduate students, post-doctoral fellows and established researchers, and the facilitation of mobility through more flexible visa arrangements, perhaps modeled on the Asia-Pacific Economic Cooperation (APEC) business travel card, which allows for pre-cleared multiple entry for frequent business travelers within the APEC region. Of course, there is still much to learn about the complex processes of scientific discovery, innovation, and the role of collaboration. These issues are of particular moment in the Asia-Pacific because of the fast emergence of new players with dramatically increased capacity for discovery. We encourage further collective thought and suggest that research organizations, think tanks, and universities in the region would do well to elaborate shared programs to better understand the various existing mechanisms that promote scientific collaboration.

\section{References}

1. Royal Society, Knowledge, Networks and Nations: Global Scientific Collaboration in the 21st Century (RS Policy document 03/11, Royal Society, London, 2011); http:// royalsociety.org/policy/projects/knowledge-networksnations/report/.

2. B. F. Jones, S. Wuchty, B. Uzzi, Science 322, 1259 (2008).

3. F. Havemann, M. Heinz, H. Kretschmer, J. Biomed. Discov. Collab. 1, 6 (2006).

4. M. Porter, Econ. Dev. Q. 14, 15 (2000).

5. C. Lee, W. F. Miller, M. G. Hancock, H. S. Rowen, Eds., The Silicon Valley Edge: A Habitat for Innovation and Entrepreneurship (Stanford Univ. Press, Stanford, CA, 2000).

6. P. Judge, Businessweek, 25 August 1997; www.businessweek.com/1997/34/b354197.htm (1997)

7. Economist, 2 September 2010; www.economist.com/ node/ 16945043

8. K. Fujita, R. C. Hill, Innovative Tokyo (World Bank Policy Research Working Paper 3507, World Bank, Washington,DC,2005); http://papers.ssrn.com/sol3/ papers.cfm?abstract_id=660088).

9. OECD, National Innovation Systems (OECD Publications, Paris, 1997).

10.1126/science. 1208378 